OPEN ACCESS

Edited by:

Raj Sengupta,

Royal National Hospital for Rheumatic

Diseases, United Kingdom

Reviewed by:

Jose Inciarte-Mundo,

Hospital Clínic de Barcelona, Spain

Arti Mahto,

King's College Hospital NHS

Foundation Trust, United Kingdom

${ }^{*}$ Correspondence:

Laurence Duquenne

I.duquenne@/eeds.ac.uk

${ }^{\dagger}$ These authors share first authorship

Specialty section: This article was submitted to Rheumatology,

a section of the journa

Frontiers in Medicine

Received: 27 July 2020 Accepted: 12 October 2020 Published: 30 October 2020

Citation:

Duquenne L, Chowdhury R, Mankia K and Emery P (2020) The Role of

Ultrasound Across the Inflammatory

Arthritis Continuum: Focus on

"At-Risk" Individuals.

Front. Med. 7:587827.

doi: 10.3389/fmed.2020.587827

\section{The Role of Ultrasound Across the Inflammatory Arthritis Continuum: Focus on "At-Risk" Individuals}

\author{
Laurence Duquenne ${ }^{1,2 *}$, Rahaymin Chowdhury ${ }^{3 \dagger}$, Kulveer Mankia ${ }^{1,2}$ and Paul Emery ${ }^{1,2}$ \\ ${ }^{1}$ Leeds Biomedical Research Centre-NIHR, Leeds, United Kingdom, ${ }^{2}$ Leeds Institute of Rheumatic and Musculoskeletal \\ Medicine, University of Leeds, Leeds, United Kingdom, ${ }^{3}$ Leeds Teaching Hospital Trust, Leeds, United Kingdom
}

In individuals at-risk of developing inflammatory arthritis, the value of an ultrasound (US) scan assessment to predict progression has been demonstrated repeatedly. However, depending on recruitment criteria, these individuals may be at different stages in the arthritis development continuum, therefore representing a heterogeneous population. As a consequence, the predictive value of ultrasound results may differ between cohorts. As other reviews have focused on the challenges in population recruitment or have combined biomarkers predicting value according to one recruitment pathway, we wanted to focus on the sole use of ultrasound assessment and its variation according to population recruitment criteria. In this review, we discuss the use of ultrasound in the different at-risk populations across the inflammatory arthritis disease continuum. This review demonstrates that although some sub-population data is scarce, ultrasound is best predictive in three at-risk populations: those with a positive ACPA test in the context of non-specific MSK symptoms, those with clinically suspect arthralgia and those with palindromic rheumatism. We consider that ultrasound assessment will be a cornerstone in prediction risk modeling and prevention studies of the preclinical phases of IA in the future.

Keywords: arthritis (including rheumatoid arthritis), ultrasound, at risk, prediction, ACPA, anti-cyclic citrullinated peptide antibodies

\section{INTRODUCTION}

Since the availability of biologic therapies, rheumatology practice has entered a new era where achieving damage free remission in rheumatoid arthritis (RA) is not only feasible, but common. As early treatment decreases long term joint damage and impaired quality of life, much effort has been made to refer, diagnose and treat patients early (1). We are now evolving toward the next stage of inflammatory arthritis management: prevention. The new priority is to identify individuals that might eventually develop inflammatory arthritis (IA): the "At-risk" individuals and treat them before arthritis occurs thereby preventing progression to RA. At-risk individuals with subclinical inflammation are at increased risk of arthritis development. It is therefore logical to suggest that this population should be considered for treatment, therefore should be included in the "window of opportunity" (2).

One of the many challenges is that arthritis development is a late step in a long process sometimes called "the inflammatory arthritis disease continuum" (3), where the preclinical phase 
includes, genetic, environmental, and systemic factors which may arise years before arthritis occurs $(4,5)$. When recruited into research cohorts, at-risk individuals might be at different stages of the continuum therefore representing heterogeneous populations, with differing risks of progression to IA.

In this review, we discuss the use of ultrasound (US) in the different at-risk populations across the arthritis disease continuum (see Figure 1). The populations in which US may be most informative will be discussed. We included peer-reviewed, published research including, retrospective and prospective analyses, observational, and interventional studies that were relevant for research and clinical practice. Only articles in English language were included.

\section{US Findings in Healthy Subjects}

As the aim of this review is to describe the evidence of US abnormalities in the preclinical phase of IA, it seems important to first consider their prevalence in healthy subjects. A first analysis found a power Doppler (PD) signal in $11 \%$ of hands and wrists of 27 healthy volunteers, especially in the wrists (6). Using the OMERACT consensus, analysis of 127 healthy controls (HC) matched with another 127 patients with early arthritis from the ESPOIR cohort found that $11 \%$ of the MCP joints 2-4 and fifth MTP joints analyzed had bone erosions (BE), 22\% had synovial hypertrophy $(\mathrm{SH}) \geq$ grade 1 and $9 \% \geq$ grade $2(7)$. Another study showed high prevalence of SH grade 1 in healthy subjects (15\%) as well as in RA patients (56\%), and no association with PD, tenderness or swelling, suggesting that only Grade $\geq 2$ should be considered pathological (8). This is also suggested by a analysis on 46 young healthy subject who showed SE in almost $20 \%$ of them (9).

The most comprehensive analysis included 32 joints of 207 HC subjects (6,621 joints in total) (10), showing that the prevalence of US abnormalities was low at joint level but high at individual level 9 vs. 88\%), and most commonly synovial effusion (SE) (69\% of joints with an abnormality). BE were found only in first metatarso-phalangeal (MTP1) joints $(n=4)$ and always with PD. The most prevalent joints with US findings were the MTP1 joints followed by MTP joints 2 to 5, then wrists, metacarpo-phalangeal (MCP) joints (especially the third), and finally proximal inter-phalangeal (PIP) joints which were almost never involved. Grade 1 was the most commonly score found, higher grades were only found in feet (10). Grade 1 SE and SH were highly prevalent in healthy subjects, the authors suggested excluding these parameters from the OMERACT ultrasound protocols $(7,10)$. None of the analyses showed differences between men and women. Only one study showed significant effect of age, especially in the feet (10). While all of the above studies used semi-quantitative measurement, another analysis of 78 individuals determined quantitative measurement of the radio-carpal abnormalities with a greater chance to indicate RA (11).

Taken together the above studies suggest that the presence of low grade US abnormalities (especially grade $1 \mathrm{SE}$ and $\mathrm{SH}$ and feet localization) are often present and therefore should not be considered pathognomonic for inflammatory arthritis. It is interesting to notice differences between each MTP, the first and fifth showing the most abnormalities and that larger joints such as elbows, ankles and shoulders have not been analyzed. Also, midfoot joints are not considered in any of the studies. The EULAR-OMERACT combined score defined synovitis as both $\mathrm{PD}$ and $\mathrm{GS} \geq 1$ (GS including $\mathrm{SH}$ and $\mathrm{SE}$ ), including only small joints, with no difference of scoring between joints (12).

\section{The Use of US in First Degree Relatives of Patients With RA}

Because a family history of RA has been shown to increase the risk of developing this disease, the use of

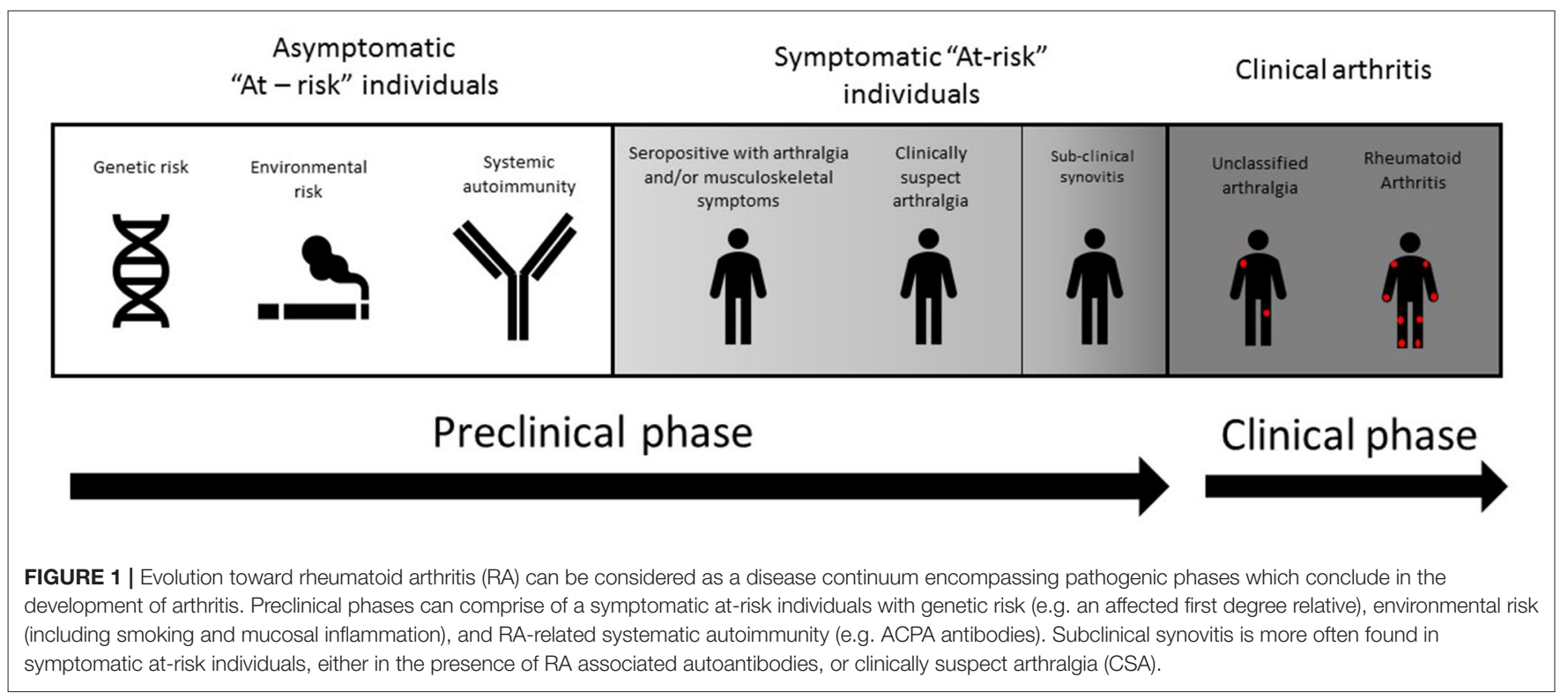


US as a prediction tool for the development of arthritis in first degree relatives (FDRs) of RA probands has been investigated.

A small study of 20 patients with RA and 25 of their FDRs was undertaken to explore the presence of abnormal US findings in FDRs without clinical arthritis $(13,14)$. Eight FDRs had arthralgia symptoms, but all were negative for RF and ACPA. The study confirmed the presence on US of inflammatory activity in FDRs (10/25 patients, $40 \%)$ and offers support for the use of US as a screening tool in this at-risk population. This study was limited by the small number of participants and did not present the US findings in detail.

A large prospective study investigated a cohort of 237 FDRs of RA patients (15). The population included a spread across the spectrum of RA development and was classified into four groups (three preclinical and one clinical). The first group were those "without risk factors," meaning those negative for the shared epitope, anti-citrullinated protein antibody (ACPA) or rheumatoid factor (RF) and had no symptoms of possible RA ( $n$ $=45$ ). The second group had risk factors, with either presence of one or two copies of the shared epitope or an ACPA positive test but no symptoms associated with possible RA $(n=38)$. The third group included subjects with inflammatory arthralgia, or self-reported symptoms associated with possible RA $(n=$ 132) and the fourth group had features of unclassified arthritis (UA) $(n=58)$. The authors found that active US findings were associated with the presence of UA on examination but not with the earlier preclinical phases of RA development, including those who had genetic risk factors but were asymptomatic. There was no statistical significance between the US results of ACPA positive and negative FDRs, however it is worth noting that the US scores for these groups were quite low, [mean B-mode score (SD): 6.7 (3.6) vs 6.8 (3.6)], OR: 1.0 (95\% CI: 0.9 to 1.1 ); mean Doppler score (SD): 0.8 (1.3) vs. 1.2 (1.9), OR: 1.2 [95\% CI: (0.9 to 1.6]). In addition, there were no demographic or clinical risk factors significantly associated with active US findings except for older age.

These results do not support a role for US in FDRs without symptoms, as part of a screening strategy for preclinical RA detection in FDRs, with the possible exception of those with UA features. Further analysis on the individuals who are positive with the shared epitope and/or an ACPA tests would improve categorizing risk populations.

\section{The Use of US in Individuals With Clinically Suspect Arthralgia}

Other studies have explored the use of ultrasound in predicting IA in patients with clinically suspect arthralgia (CSA). CSA was defined by EULAR as a set of characteristics to be used in patients with arthralgia without clinical arthritis and without other diagnosis or explanation for the arthralgia (16).

Although many of the following studies were performed prior to this definition, they do follow the same theme of including patients who had inflammatory arthralgia but had no clinical synovitis (CS). An example of this is a large multicentre study which included patients who presented with at least two painful joints but no CS, with symptoms that lasted less than a year (17). Of the 196 patients who were included, 159 patients completed follow up over a 1 year period, only $15 \%$ were ACPA positive. The authors defined US synovitis as GS $\geq 2$ and $\mathrm{PD} \geq 1$ and reported a statistically significant association between US synovitis and prediction of IA (OR 3.03, 95\% [CI: 1.69-5.41]). They concluded that the lack of US synovitis was a strong negative predictor for IA. These findings were further supported by a retrospective analysis of 80 consecutive patients using SONAR B-mode criteria to determine significant synovitis $(18,19)$. They found that significant US GS synovitis appeared to be the only independent predictor of RA on multivariate analysis (OR 7.4 [95\%CI: 1.1942.8]) in ACPA negative patients who presented with polyarthralgia and no CS.

A recent study compared the US and MRI results of 70 individuals with CSA $(n=40)$ and early IA $(n=30)$. They showed an overall significant correlation between both imaging techniques regarding synovitis and tenosynovitis, especially in the MCP and wrists joints, although MRI was more sensitive. Although less frequently present in the CSA subgroup, similar results of concordance were found, but with a lower sensitivity (20).

Thus, the literature would suggest that US does add additional value to clinical and laboratory investigations in predicting IA in those with CSA. However, there are a paucity of data on US as MRI has been preferred and further US investigation is recommended.

\section{The Use of US in ACPA (and/or RF) Positive Individuals With Musculoskeletal Symptoms}

There have been various studies on the use of US to predict RA in at-risk populations who have been selected on the base of an ACPA and/or RF positive, with MSK symptoms (including arthralgia), but no CS.

An important observational cohort study investigating US as a predictor for IA in ACPA positive at-risk individuals was conducted by a group in Leeds (21). The 100 consecutive participants included were ACPA positive, had a new nonspecific musculoskeletal (MSK) symptom, but no CS. They demonstrated in multivariable analysis a significant association between PD at the patient level and the development of IA (HR 1.88 [95\% CI 1.07-3.29]) and incorporated it in a prediction model including serological and clinical measures. In Amsterdam, a prospective cohort study of 192 participants who were ACPA and/or RF positive, found that Gray Scale (GS) and PD were predictive for IA at the joint level, but this did not reach statistical significance at the patient level, meaning that there was a significance in US findings predicting which joint would progress to clinical arthritis, but not which patients would progress to IA (22). It is useful to note that US protocols varied between both studies; while Amsterdam's US protocol included only painful, adjacent, and contralateral joints if hands were involved, the Leeds study included all MCP joints, PIP joints and both wrists. A follow up study in Leeds included 136 individuals and added MTP joints to the analysis (23), they concluded that all US findings ( $\mathrm{BE}$ grade $\geq 1$, GS grade $\geq 2$ and $\mathrm{PD}$ grade $\geq 1$ ) 
could predict progression to IA and its timing, with the risk being greatest in those patients with at least one joint with PD signal on US. Unlike the Amsterdam cohort, the predictive value of PD was significant at both the joint and patient level. The discrepancy in findings may have been due to the differing inclusion criteria in the studies. While the Amsterdam cohort included about a third of RF positive-ACPA negative individuals, Leeds group included only ACPA positivity, suggesting that individuals in the two cohorts could have been at different level of progression risk. Additionally, the US protocols in both studies were different, Leeds scanned 32 joints including bilateral wrists, MCPs, PIPs and MTPs. Both studies were amongst the first to include only patients without CS.

A follow up prospective cohort study from Amsterdam (24) included a cohort of 163 seropositive (RF and/or ACPA) patients who had arthralgia but no CS in a prospective cohort. After excluding metatarsophalangeal joints (MTPs), they showed that GS had a significant predictive value to progression to IA (OR 6.6 [95\%CI 1.9-22]). Unlike the other above studies, PD was not found to be predictive in this study. The authors attributed this main difference to the alternative scanning protocol and technical differences in US machines. Differences can also be ascribed to the inclusion criteria, as Nam et al. included ACPA positive patients only (18).

In two further analyses, the first (25) analyzed US images from 319 patients and found that the number of joints with PD or tenosynovitis (TSV) was predictive of progression to IA with high specificity and moderate sensitivity with respective hazard ratios of $1.2(p=0.026)$ and $1.13(p=0.025)$, the addition of ACPA titer improved the predictive value of the number of joints with PD with a specificity/sensitivity of $0.92 / 0.34$ (AUC 0.964). The authors also suggested that a selection of jointsmainly in the hands and feet-with better predictive power could improve US sensitivity. Another multivariable analysis on the same population $(n=488)$ showed that individuals with $1-$ 3 joints with a PD signal or 1-2 with $\mathrm{BE}$ were twice as likely to develop IA, those with $\geq 4$ joints with a $\mathrm{PD}$ signal were more than six times more likely (26). A more recent study (27) analyzed baseline US scans of a further 419 CCP positive at-risk individuals from the Leeds CCP cohort. In this analysis, the most predictive features for the development of clinical arthritis on US were $\mathrm{BE}$ in $>1$ joint or BE combined with synovitis in the MTP5 joint (OR 10.6 [95\% CI 1.9 to 60.4] $p<0.01$ ) and 5.1 [95\% CI 1.4 to 18.9 ] $p=0.02$ ] respectively. While presence of $\mathrm{BE}$ in any joint was previously described predictive of progression to IA (23), this study suggests that some joints might be more specific for progression.

Overall, the discrepancies in some of the findings are likely due to population variability: whether selected individuals are tested positive for RF and/or ACPA, associated symptoms and set of joints analyzed, but also the factors around how to perform the US itself, defining US synovitis, the optimum number and specific joints to be incorporated in the US protocol and the use of different scoring systems.

The above studies demonstrate that there is strong evidence that all US features including GS, BE, TSV, and especially PD presence, have an important part to play in predicting IA in seropositive at-risk individuals with MSK symptoms. They also discussed scanning protocols, while a limited, focused joint set can be used to identify erosions and predict arthritis; pragmatic scanning protocol could be easily incorporated into clinical practice. Ideally future studies should use standardized US protocols and scoring systems.

\section{The Use of US in Individuals With Palindromic Rheumatism}

Some individuals-ACPA positive or not-present with intermittent inflammatory flares, alternating between short attacks of pain and swelling and asymptomatic periods. Specific clinical and US features found in these individuals suggest that palindromic rheumatism (PR) could be a discrete pathological entity (28). However, shared risk factors with RA suggest PR could also be considered a phase of the RA continuum (3). Different studies have focused on this population, whether it be during or in-between flares of joint symptoms. In all these analyses, the proportion of ACPA positive patients ranges between 13 and $66.7 \%$.

In 2014, a group analyzed 11 joints in the hands of 54 patients outside of a flare (29). The joints reported to be involved in the first flare varied according to ACPA status, with an increased ratio of small joints involvement in ACPA positive participants. This antibody based discrepancy was confirmed on US performed between flares. At the joint level, they found that only $2.8 \%$ of the 1,188 joints analyzed had $\mathrm{SH} \geq 2$, with $\mathrm{PD} \geq 1$ in $1.4 \%$ of these, mostly wrists and MCP. At the patient level, 25.9\% of them presented US synovitis in at least one joint $(\mathrm{SH} \geq 2$, or $\mathrm{PD} \geq 1$ ). Patients in this cohort had long disease duration (mean duration of 11.6 years) and $61.1 \%$ were on DMARDs (mainly hydroxychloroquine). Ten patients of this study were also assessed during a flare, none of them showed periarticular US abnormalities, seven showed an intra-articular PD signal.

In a small cohort $(n=15)$, analysis of US scans taken during flares in the hands and wrists showed US synovitis in $60 \%$ of individuals $(n=9 / 15)$, with PD in 6 of them (30). The largest analysis to date was based on $84 \mathrm{PR}$ patients during a flare (31). While $78 \%$ of the participants had signs of PD presence, only $31 \%$ of these were intra-articular PD, the rest were features of TSV and/or periarticular soft tissue inflammation. Moreover, Intraarticular PD presence and ACPA positivity were both recognized as predictors of progression to RA (respectively: $\mathrm{OR}=2.28$ [95\%CI: 0.67-7.68] and OR = 6.18 [95\% CI: 1.50-25.52]).

The most recent imaging analysis in $\mathrm{PR}$ was performed on 79 individuals comparing US and MRI (32). This analysis was the first to include treatment-naïve individuals, with a short duration of symptoms. The authors compared US examinations taken during and between flares, and showed US of the flaring area showed significantly more TSV (23 vs. $4 \%$ ), more signs of extracapsular inflammation (61 vs. 15\%), particularly periarticular inflammation (39 vs. 4\%), (and non-significantly different PD synovitis [23 vs. $7 \%]$ ) than in-between flares. Interestingly, there was no influence of the antibody status on the US features. These results suggest that palindromic rheumatism has a discrete imaging phenotype and that some features such as ACPA positivity and intra-articular inflammation during a flare may increase the risk of developing RA. 
These studies all included individuals with PR, which US has revealed some specific features such as higher proportion of extra-capsular abnormalities compared to at-risk and RA individuals. Discrepancies can be explained by recruitment criteria differences such as the use of previous medication, proportion of ACPA positive individuals and symptoms duration. Two analyses showed predominance of US extraarticular inflammation while all showed presence of subclinical inflammation.

\section{Ultrasound in Undifferentiated Arthritis}

Early diagnosis is a priority, it is therefore essential to improve diagnostic success rates at first referral. Because of its power and accessibility, US is now recognized as an essential tool for IA diagnosis and management. We have discussed US findings and implication in individuals with no CS (the at-risk populations), individuals with intermittent CS (palindromic rheumatism). Here we discuss how US use has been a major tool in assessing patients at CS onset. This chapter will not focus on RA patients but those with undifferentiated arthritis (UA) who are presenting with early CS but do not meet classification criteria for a specific rheumatic disease."

Many studies have shown US superiority to clinical examination in detecting synovitis (33-35). In a cohort of 50 ACPA and RF negative patients with UA from early arthritis clinics, they showed that IA probability increased from 6 to $8-85 \%$ depending on which two US features were present (GS = $3, \mathrm{PD} \geq 1$ or $\mathrm{BE}$ presence) (36). This was confirmed in a large study $(n=831)$ where $31 \%$ of patients progressed to persistent IA, baseline serological and clinical biomarkers were already predictive of progression, but US improved all predictive values, particularly in the seronegative patients (AUC increase of $9 \%, p$ $<0.001$ ) (37). Another study focused on EULAR classification criteria for RA, with the same recruitment criteria $(n=109)$, $61 \%$ of participants presented with a swollen joint, $30 \%$ were $\mathrm{ACPA}+$ (compared to $15.4 \%$ in the previous study). They showed that $G S \geq 1$ improved the sensitivity of the 2010 criteria from 58 up to $78 \%$ without decreasing specificity (AUC 0.868), which was $93.7 \%$ if $\mathrm{GS} \geq 2$ or $\mathrm{PD} \geq 1$ were present, but at the price of decreased specificity which went down to $56.1 \%$ (AUC 0.844 ) (38). US was also shown to be especially useful in RA diagnosis in CCP negative very early UA. Indeed, in a study recruiting only CCP negative individuals with suspected UA, US synovitis significantly improved the sensitivity of the 2010 classification criteria up to $86.2 \%$ (39), meaning that US could counterbalance the absence of specific serology findings. While using a probabilistic approach depending on the practitioner's impression, another group showed that the addition of US to routine investigations increased the diagnostic certainty of UA from 31.1 to $61.2 \%(p<0.001)(40)$. It is interesting that in these studies, more than half of patients presented abnormalities on first US but not all developed a persistent disease, suggesting that, focusing on the most specific US features such as PD presence and/or GS $\geq 2$ might improve US accuracy. Another study showed that GS was more effective at showing synovitis than clinical examination, laboratory investigation $(p=0.00015)$, and plain film radiography ( $p=0.0002)$ (41). In a study recruiting individuals with at least one swollen joint, the same improvement of the AUC for RA diagnosis was found, they also showed that MCP joints were highly specific for IA (42). Others found that selecting PD grade 2 increased discriminative ability (43).

Interestingly, in a large $(n=379)$ retrospective analysis on a cohort of patients referred to early arthritis clinic and followed for minimum 12 months, US parameters did not show significant predictive value for persistent IA in comparison to clinical parameters alone, (AUC curve both metrics: 0.91; [95\% CI $0.89-0.94$ ] [95\% CI 0.88 to 0.94 ] respectively) (44). This same group did a further study with different scanning protocol and comparison methodology between clinical and US variables, which did show improvement of predictive values when comparing to clinical parameters alone (37).

The same team developed two "risk metrics" computerized tools using logistic regression to predict the development of persistent IA whereas the first models used multivariate and ROC curve analysis to identify discriminators of IA and the added value of US parameters (44). Another diagnostic model for progression from UA to RA was designed combining symptoms and morning stiffness durations, raised inflammatory markers, CCP and or RF positivity and PDUS presence in 1,2 or $\geq 3$ joints, which provided an impressive AUC of $0.919(n=149)(45)$.

After a RA diagnosis has been made, US has also shown a good correlation with composite scores of disease activity at all points of disease evolution, as well as with radiological damage $(33,46-48)$. It is suggested for use in the assessment of remission, prediction of flares, and to assess risk of relapse when tapering treatment as well as to inform the need to intensify treatment (49-55). PROMPT trial randomized $110 \mathrm{UA}$ individuals to receive methotrexate (MTX) vs. placebo for 1 year depending on US results. At 5 years, they showed no difference in progression rates to $\mathrm{RA}$, only a delay in progression in the treated ACPA positive participants (56).

Overall, US has proved its place in diagnosis, disease activity and remission assessment while, for now, US driven trials have shown variable results.

\section{DISCUSSION}

The main challenge in populations at-risk of RA is to categorize biomarkers that are specific, sensitive, and reproducible in predicting disease progression. Some of them may be present years before progression and remain stable-such as specific antibodies or genetic predisposition-whilst others may vary with time or only appear closer to the clinical phase of the disease such as abnormalities on high-resolution imaging, for example, US. Defining the phase where sub-clinical inflammation on imaging appears is particularly important, as it represents the initial onset of articular inflammation and as such, the phase where clinical arthritis is imminent. Indeed, as one RCT has shown to delay the onset of RA (57), the preclinical phase of RA could be the optimal time point to initiate treatment as damages have not occurred yet, thus re-defining the window of opportunity. Delaying or even preventing the onset of RA will have major social, financial, and personal impact on patients and society.

Retrospective studies on RA patients were highly useful initially to find out biomarkers that were present before 
symptoms, but these are limited in the quantity and quality of the data. For example, not all analysis was possible on frozen blood samples, no imaging was performed, and clinical data were absent. Therefore, observational studies on RA prediction should now be all prospective. Another important challenge in at-risk population studies are the discrepancies between and within cohorts depending on the recruitment criteria. This implies that results are difficult to replicate and/or compare between populations. We discussed above the various pathways of prospective recruitment, for example, CSA and ACPA+ individuals, with a usual progression to IA rate of around one third of participants. This low progression rate increases duration of follow-up needed, tests and visits to be repeated, and the need for large cohorts to get significant results. Only a few centers are able to support this.

Identifying individuals who would benefit the most from an US assessment is of major importance. Indeed, although US sub-clinical inflammation can be found throughout the whole disease continuum toward RA, US abnormalities have shownat present-only of predictive value for disease development in specific populations. The most representative are symptomatic at-risk individuals who have been identified by antibody positivity while in some populations, for example, in FDRs and CSA individuals, data are sparse and would benefit from further study. Another limitation is that not all analysis used the same US protocols. Even if we nowadays tend to follow EULAR/OMERACT recommendation, this has not always been the case. Although some US protocols focusing on specific joint sets are suggested in RA to improve US pragmatic use in clinic $(58,59)$, no joint based analysis have been performed on at-risk individuals yet. Nevertheless, focus is often on the small joints such as MCP, PIP, MTP, and wrists.

The studies investigating the role of US in FDRs do not support its use in those without symptoms. There is however a paucity of data in this area and further exploration is needed. On the other hand, the predictive value of US for IA development was greater in the individuals with MSK symptoms identified by a positive ACPA test. In this group particularly, depending on the US scan protocols and the recruitment criteria, US features have shown significant predictive value at the joint and patient level, for GS, BE, TSV, and especially for PD presence. This is more consistent if we consider the studies of individuals with more stringent inclusion criteria, with cohorts more likely to be at imminent risk of progression. In individuals with CSA, MRI-US comparison has shown good correlation, mainly for specificity. At diagnosis of early UA, some studies showed US superiority to clinical examination, efficacy in RA diagnosis, disease activity assessment, and treatment efficacy. In this population, efficacy is not dependent on the serological results and might be of more value in the seronegative individuals. It has good discriminative value, improving the classification criteria's sensitivity. In established RA, although it has been shown to be a good predictor in treatment response, remission assessment, and flare prediction, two trials comparing conventional T2 $\mathrm{T}$ approach with US lead approach did not show significant differences in DAS28 remission, and lead to an increased treatment regimen is the US groups $(60,61)$. Nevertheless, secondary analysis showed that Boolean remission was more often reached in the US arm (61).

At present without guidance, rheumatologists have different approaches to managing at-risk individuals. A survey conducted in 2019 across the UK showed that $73 \%$ of practitioners would treat ACPA positive individuals if at least one joint showed PD presence on US (62). This reflects the pragmatic approach used due to the lack evidence on which treatment is the most appropriate, which population would respond well and what is the most appropriate timing to start treatment. This lack of global consensus emphasizes the need for research studies to assess these questions (63). Although individuals followed in preventive observational cohorts showed milder disease activity at progression (64), long term impact of prevention clinics have not been assessed yet. A few randomized controlled trials have been designed on individuals without CS. While one used the presence of US inflammation as part of the recruitment criteria (57), another one collected US data along the study for secondary analysis, results are not published yet (65), none of the others included US as an outcome or collected longitudinal data (66-68). Some RA treatments have been tested on individuals with UA (56) or even before CS occurs (65), the complexity here is to define the optimal high risk individuals who may benefit from treatment as well as the participant acceptance for a medication without confirmed disease $(69,70)$. At present, no formal economic analyses for use of US or treatment in atrisk individuals have been conducted, it therefore represents an important area for future work.

All aspects of US findings throughout the RA continuum have shown its high predictive value for progression to clinical synovitis, perhaps with PD standing out to be most predictive. However, it is difficult to compare these aspects due to the different definitions of US synovitis and scanning protocols through the studies. Overall, US does offer clear assistance in identifying sub-clinical inflammation in individuals at-risk of IA. However, we have to consider the time and resources needed for systemic prevention to be put in place. All populations considered, it appears that the greatest impact on IA prediction of US examination can be found in three at-risk populations: those with a positive ACPA test in the context of non-specific MSK symptom, those with CSA, and those with palindromic rheumatism. Since it has shown such good predictive value in IA and in the preclinical phases of IA, it is expected that US will be a cornerstone in prediction risk modeling and prevention studies. Nonetheless, further studies with unified selection criteria, specific joints and/or feature selections are still needed to improve US impact relevance.

\section{AUTHOR CONTRIBUTIONS}

LD and RC have participated with equal contribution and would like to share first authorship. KM: important part in topic choices, discussion, proof reading, and time input. PE: topic choices, discussion, and final approval for submission. All authors contributed to the article and approved the submitted version. 


\section{REFERENCES}

1. Nell VP, Machold KP, Eberl G, Stamm TA, Uffmann M, Smolen JS. Benefit of very early referral and very early therapy with disease-modifying antirheumatic drugs in patients with early rheumatoid arthritis. Rheumatology. (2004) 43:906-14. doi: 10.1093/rheumatology/keh199

2. Burgers LE, Raza K, van der Helm-van Mil AH. Window of opportunity in rheumatoid arthritis-definitions and supporting evidence: from old to new perspectives. RMD Open. (2019) 5:e000870. doi: 10.1136/rmdopen-2018-000870

3. Mankia K, Emery P. Palindromic rheumatism as part of the rheumatoid arthritis continuum. Nat Rev Rheumatol. (2019) 15:687-95. doi: 10.1038/s41584-019-0308-5

4. Nielen MM, van Schaardenburg D, Reesink HW, van de Stadt RJ, van der Horst-Bruinsma IE, de Koning MH, et al. Specific autoantibodies precede the symptoms of rheumatoid arthritis: a study of serial measurements in blood donors. Arthritis Rheum. (2004) 50:380-6. doi: 10.1002/art.20018

5. Silman AJ, Hennessy E, Ollier B. Incidence of rheumatoid arthritis in a genetically predisposed population. Brit J Rheumatol. (1992) 31:365-8. doi: 10.1093/rheumatology/31.6.365

6. Terslev L, Torp-Pedersen S, Qvistgaard E, von der Recke P, Bliddal H. Doppler ultrasound findings in healthy wrists and finger joints. Ann Rheum Dis. (2004) 63:644-8. doi: 10.1136/ard.2003.009548

7. Millot F, Clavel G, Etchepare F, Gandjbakhch F, Grados F, Saraux A, et al. Musculoskeletal ultrasonography in healthy subjects and ultrasound criteria for early arthritis (the ESPOIR cohort). J Rheumatol. (2011) 38:613-20. doi: 10.3899/jrheum.100379

8. Witt M, Mueller F, Nigg A, Reindl C, Leipe J, Proft F, et al. Relevance of grade 1 gray-scale ultrasound findings in wrists and small joints to the assessment of subclinical synovitis in rheumatoid arthritis. Arthritis Rheum. (2013) 65:1694-701. doi: 10.1002/art.37954

9. Rosenberg C, Arrestier S, Etchepare F, Fautrel B, Rozenberg S, Bourgeois P. High frequency of ultrasonographic effusion in interphalangeal joints of healthy subjects: a descriptive study. Joint Bone Spine. (2009) 76:265-7. doi: 10.1016/j.jbspin.2008.06.017

10. Padovano I, Costantino F, Breban M, D'Agostino MA. Prevalence of ultrasound synovial inflammatory findings in healthy subjects. Ann Rheum Dis. (2016) 75:1819-23. doi: 10.1136/annrheumdis-2015-208103

11. Machado FS, Furtado RN, Takahashi RD, de Buosi AL, Natour J. Sonographic cutoff values for detection of abnormalities in small, medium and large joints: a comparative study between patients with rheumatoid arthritis and healthy volunteers. Ultrasound Med Biol. (2015) 41:989-98. doi: 10.1016/j.ultrasmedbio.2014.12.004

12. D’Agostino MA, Terslev L, Aegerter P, Backhaus M, Balint P, Bruyn GA, et al. Scoring ultrasound synovitis in rheumatoid arthritis: a EULAROMERACT ultrasound taskforce-Part 1: definition and development of a standardised, consensus-based scoring system. RMD Open. (2017) 3:e000428. doi: 10.1136/rmdopen-2016-000428

13. Soliman E. SAT0650 Musculoskeletal ultrasound in first degree relatives of rheumatoid arthritis patients. Ann Rheum Dis. (2018) 77(Suppl. 2):1175. doi: 10.1136/annrheumdis-2018-eular.2349

14. Soliman E, Ohrndorf S, Zehairy M, Matrawy K, Alhadidy A, Abdelati A. Osteopontin, osteoprotegerin and musculoskeletal ultrasound findings in first-degree relatives of rheumatoid arthritis: potential markers of preclinical disease. Preprint. (2020). doi: 10.21203/rs.3.rs-20512/v1

15. Brulhart L, Alpízar-Rodríguez D, Nissen MS, Zufferey P, Ciubotariu I, Fleury G, et al. Ultrasound is not associated with the presence of systemic autoimmunity or symptoms in individuals at-risk for rheumatoid arthritis. RMD Open. (2019) 5:e000922. doi: 10.1136/rmdopen-2019-0 00922

16. van Steenbergen HW, Aletaha D, Beaart-van de Voorde LJ, Brouwer E, Codreanu C, Combe B, et al. Eular definition of arthralgia suspicious for progression to rheumatoid arthritis. Ann Rheum Dis. (2017) 76:491-6. doi: 10.1136/annrheumdis-2016-209846

17. van der Ven M, van der Veer-Meerkerk M, Ten Cate DF, Rasappu N, Kok MR, Csakvari D, et al. Absence of ultrasound inflammation in patients presenting with arthralgia rules out the development of arthritis. Arthritis Res Ther. (2017) 19:202. doi: 10.1186/s13075-017-1405-y
18. Zufferey P, Rebell C, Benaim C, Ziswiler HR, Dumusc A, So A. Ultrasound can be useful to predict an evolution towards rheumatoid arthritis in patients with inflammatory polyarthralgia without anticitrullinated antibodies. Joint, Bone, Spine. (2017) 84:299-303. doi: 10.1016/j.jbspin.2016.05.011

19. Zufferey P, Brulhart L, Tamborrini G, Finckh A, Scherer A, Moller B, et al. Ultrasound evaluation of synovitis in RA: correlation with clinical disease activity and sensitivity to change in an observational cohort study. Joint Bone Spine. (2014) 81:222-7. doi: 10.1016/j.jbspin.2013.08.006

20. Ohrndorf S, Boer AC, Boeters DM, Ten Brinck RM, Burmester GR, Kortekaas $\mathrm{MC}$, et al. Do musculoskeletal ultrasound and magnetic resonance imaging identify synovitis and tenosynovitis at the same joints and tendons? A comparative study in early inflammatory arthritis and clinically suspect arthralgia. Arthritis Res Ther. (2019) 21:59. doi: 10.1186/s13075-019-1824-z

21. Rakieh C, Nam JL, Hunt L, Hensor EM, Das S, Bissell LA, et al. Predicting the development of clinical arthritis in anti-CCP positive individuals with nonspecific musculoskeletal symptoms: a prospective observational cohort study. Ann Rheum Dis. (2015) 74:1659-66. doi: 10.1136/annrheumdis-2014-205227

22. van de Stadt LA, Bos WH, Meursinge Reynders M, Wieringa $H$, Turkstra $F$ van der Laken CJ, et al. The value of ultrasonography in predicting arthritis in auto-antibody positive arthralgia patients: a prospective cohort study. Arthritis Res Ther. (2010) 12:R98. doi: 10.1186/ar3028

23. Nam JL, Hensor EM, Hunt L, Conaghan PG, Wakefield RJ, Emery P. Ultrasound findings predict progression to inflammatory arthritis in anti-CCP antibody-positive patients without clinical synovitis. Ann Rheum Dis. (2016) 75:2060-7. doi: 10.1136/annrheumdis-2015-208235

24. van Beers-Tas $M H$, Blanken AB, Nielen MMJ, Turkstra F, van der Laken CJ, Meursinge Reynders M, et al. The value of joint ultrasonography in predicting arthritis in seropositive patients with arthralgia: a prospective cohort study. Arthritis Res Ther. (2018) 20:279. doi: 10.1186/s13075-018-1767-9

25. Duquenne L, Mankia K, Montoya L, Di Matteo A, Nam J, Emery P. P240 Can ultrasound alone predict the need to treat ACPA positive individuals without synovitis? Rheumatology. (2020) 59. doi: 10.1093/rheumatology/keaa111.234

26. Duquenne L. MK, Di Matteo A, Garcia-Montoya L, Nam J, Emery P. ACPA positive At-risk individuals without clinical arthritis, is ultrasound sufficiently accurate to predict progression to inflammatory arthritis? Ann Rheum Dis. (2020) 79:260

27. Di Matteo A, Mankia K, Duquenne L, Cipolletta E, Wakefield RJ, Garcia-Montoya L, et al. Ultrasound erosions in the feet best predict progression to inflammatory arthritis in anti-CCP positive at-risk individuals without clinical synovitis. Ann Rheum Dis. (2020) 79:901-7. doi: 10.1136/annrheumdis-2020-217215

28. Mankia K, Emery P. What can palindromic rheumatism tell us? Best Pract Res Clin Rheumatol. (2017) 31:90-8. doi: 10.1016/j.berh.2017.09.014

29. Cabrera-Villalba S, Ramirez J, Salvador G, Ruiz-Esquide V, Hernández MV, Inciarte-Mundo $\mathrm{J}$, et al. Is there subclinical synovitis in patients with palindromic rheumatism in the intercritical period? a clinical and ultrasonographic study according to anticitrullinated protein antibody status. J Rheumatol. (2014) 41:1650-5. doi: 10.3899/jrheum.131545

30. Bugatti S, Caporali R, Manzo A, Sakellariou G, Rossi S, Montecucco C. Ultrasonographic and MRI characterisation of the palindromic phase of rheumatoid arthritis. Ann Rheum Dis. (2012) 71:625-6. doi: 10.1136/annrheumdis-2011-200077

31. Chen HH, Lan JL, Hung GD, Chen YM, Lan HH, Chen DY. Association of ultrasonographic findings of synovitis with anti-cyclic citrullinated peptide antibodies and rheumatoid factor in patients with palindromic rheumatism during active episodes. J Ultrasound Med. (2009) 28:1193-9. doi: 10.7863/jum.2009.28.9.1193

32. Mankia K, D’Agostino MA, Wakefield RJ, Nam JL, Mahmood W, Grainger $\mathrm{AJ}$, et al. Identification of a distinct imaging phenotype may improve the management of palindromic rheumatism. Ann Rheum Dis. (2019) 78:43-50. doi: 10.1136/annrheumdis-2018-214175

33. Terslev L, von der Recke P, Torp-Pedersen S, Koenig MJ, Bliddal H. Diagnostic sensitivity and specificity of doppler ultrasound in rheumatoid arthritis. $J$ Rheumatol. (2008) 35:49-53.

34. Szkudlarek M, Court-Payen M, Strandberg C, Klarlund M, Klausen T, Ostergaard M. Power Doppler ultrasonography for assessment of synovitis in the metacarpophalangeal joints of patients with rheumatoid arthritis: a comparison with dynamic 
magnetic resonance imaging. Arthritis Rheum. (2001) 44:2018-23. doi: 10.1002/1529-0131(200109)44:9\&lt;2018::AID-ART350>3.0.CO;2-C

35. Wakefield RJ, Freeston JE, O'Connor P, Reay N, Budgen A, Hensor EM, et al. The optimal assessment of the rheumatoid arthritis hindfoot: a comparative study of clinical examination, ultrasound and high field MRI. Ann Rheum Dis. (2008) 67:1678-82. doi: 10.1136/ard.2007.079947

36. Freeston JE, Wakefield RJ, Conaghan PG, Hensor EM, Stewart SP, Emery P. A diagnostic algorithm for persistence of very early inflammatory arthritis: the utility of power Doppler ultrasound when added to conventional assessment tools. Ann Rheum Dis. (2010) 69:417-9. doi: 10.1136/ard.2008.106658

37. Iqbal K, Lendrem DW, Hargreaves B, Isaacs JD, Thompson B, Pratt AG. Routine musculoskeletal ultrasound findings impact diagnostic decisions maximally in autoantibody-seronegative early arthritis patients. Rheumatology. (2019) 58:1268-73. doi: 10.1093/rheumatology/kez008

38. Nakagomi D, Ikeda K, Okubo A, Iwamoto T, Sanayama Y, Takahashi K, et al. Ultrasound can improve the accuracy of the (2010). American College of Rheumatology/European League against rheumatism classification criteria for rheumatoid arthritis to predict the requirement for methotrexate treatment. Arthritis Rheum. (2013) 65:890-8. doi: 10.1002/art.37848

39. Ji L, Deng X, Geng Y, Song Z, Zhang Z. The additional benefit of ultrasonography to 2010 ACR/EULAR classification criteria when diagnosing rheumatoid arthritis in the absence of anti-cyclic citrullinated peptide antibodies. Clin Rheumatol. (2017) 36:261-7. doi: 10.1007/s10067-016-3465-9

40. Rezaei H, Torp-Pedersen S, af Klint E, Backheden M, Kisten Y, Gyori $\mathrm{N}$, et al. Diagnostic utility of musculoskeletal ultrasound in patients with suspected arthritis-a probabilistic approach. Arthritis Res Ther. (2014) 16:448. doi: 10.1186/s13075-014-0448-6

41. Zhang YH, Li K, Xiao J, Zhang HD, Zhang XY. Comparison of ultrasound, radiography, and clinical investigations in the diagnosis of early rheumatoid synovitis in patients with nonspecific musculoskeletal symptoms: a multicenter cross-sectional study. Med Sci Monit. (2018) 24:4372-8. doi: 10.12659/MSM.908755

42. Filer A, de Pablo P, Allen G, Nightingale P, Jordan A, Jobanputra P, et al. Utility of ultrasound joint counts in the prediction of rheumatoid arthritis in patients with very early synovitis. Ann Rheum Dis. (2011) 70:500-7. doi: 10.1136/ard.2010.131573

43. Kawashiri SY, Fujikawa K, Nishino A, Okada A, Aramaki T, Shimizu $\mathrm{T}$, et al. Ultrasound-detected bone erosion is a relapse risk factor after discontinuation of biologic disease-modifying antirheumatic drugs in patients with rheumatoid arthritis whose ultrasound power Doppler synovitis activity and clinical disease activity are well controlled. Arthritis Res Ther. (2017) 19:108. doi: 10.1186/s13075-017-1320-2

44. Pratt AG, Lorenzi AR, Wilson G, Platt PN, Isaacs JD. Predicting persistent inflammatory arthritis amongst early arthritis clinic patients in the UK: is musculoskeletal ultrasound required? Arthritis Res Ther. (2013) 15:R118. doi: 10.1186/ar4298

45. Salaffi F, Ciapetti A, Gasparini S, Carotti M, Filippucci E, Grassi W. A clinical prediction rule combining routine assessment and power Doppler ultrasonography for predicting progression to rheumatoid arthritis from early-onset undifferentiated arthritis. Clin Exp Rheumatol. (2010) 28:686-94.

46. Damjanov N, Radunovic G, Prodanovic S, Vukovic V, Milic V, Simic Pasalic $\mathrm{K}$, et al. Construct validity and reliability of ultrasound disease activity score in assessing joint inflammation in RA: comparison with DAS-28. Rheumatology. (2012) 51:120-8. doi: 10.1093/rheumatology/ker255

47. Naredo E, Möller I, Cruz A, Carmona L, Garrido J. Power Doppler ultrasonographic monitoring of response to anti-tumor necrosis factor therapy in patients with rheumatoid arthritis. Arthritis Rheum. (2008) 58:2248-56. doi: 10.1002/art.23682

48. Ribbens C, Andre B, Marcelis S, Kaye O, Mathy L, Bonnet V, et al. Rheumatoid hand joint synovitis: gray-scale and power Doppler US quantifications following anti-tumor necrosis factor-alpha treatment: pilot study. Radiology. (2003) 229:562-9. doi: 10.1148/radiol.2292020206

49. Gul HL, Eugenio G, Rabin T, Burska A, Parmar R, Wu J, et al. Defining remission in rheumatoid arthritis: does it matter to the patient? A comparison of multi-dimensional remission criteria and patient reported outcomes. Rheumatology. (2019) 59:613-21. doi: 10.1093/rheumatology/kez330
50. Paulshus Sundlisæter N, Aga AB, Olsen IC, Hammer HB, Uhlig T, van der Heijde D, et al. Clinical and ultrasound remission after 6 months of treatto-target therapy in early rheumatoid arthritis: associations to future good radiographic and physical outcomes. Ann Rheum Dis. (2018) 77:1421-5. doi: 10.1136/annrheumdis-2017-212830

51. Wang L, Geng Y, Han J, Sun X, Zhang Z. A combination model to predict relapse and successful conventional DMARDs de-escalation in rheumatoid arthritis patients with sustained clinical remission. Clin Exp Rheumatol. (2019) 37:120-6.

52. Han J, Geng Y, Deng X, Zhang Z. Subclinical synovitis assessed by ultrasound predicts flare and progressive bone erosion in rheumatoid arthritis patients with clinical remission: a systematic review and metaanalysis. J Rheumatol. (2016) 43:2010-8. doi: 10.3899/jrheum.160193

53. Geng Y, Han J, Deng X, Zhang Z. Deep clinical remission: an optimised target in the management of rheumatoid arthritis? Experience from an ultrasonography study. Clin Exp Rheumatol. (2016) 34:581-6.

54. Janta I, Valor L, De la Torre I, Martínez-Estupiñán L, Nieto JC, Ovalles-Bonilla JG, et al. Ultrasound-detected activity in rheumatoid arthritis on methotrexate therapy: which joints and tendons should be assessed to predict unstable remission? Rheumatol Int. (2016) 36:387-96. doi: 10.1007/s00296-015-3409-8

55. Han J, Geng Y, Deng X, Zhang Z. Risk factors of flare in rheumatoid arthritis patients with both clinical and ultrasonographic remission: a retrospective study from China. Clin Rheumatol. (2017) 36:1721-7. doi: 10.1007/s10067-017-3736-0

56. van Aken J, Heimans L, Gillet-van Dongen H, Visser K, Ronday HK, Speyer I, et al. Five-year outcomes of probable rheumatoid arthritis treated with methotrexate or placebo during the first year (the PROMPT study). Ann Rheum Dis. (2014) 73:396-400. doi: 10.1136/annrheumdis-2012-2 02967

57. Gerlag DM, Safy M, Maijer KI, Tang MW, Tas SW, Starmans-Kool MJF, et al. Effects of B-cell directed therapy on the preclinical stage of rheumatoid arthritis: the PRAIRI study. Ann Rheum Dis. (2019) 78:179-85. doi: 10.1136/annrheumdis-2017-212763

58. Backhaus M, Ohrndorf S, Kellner H, Strunk J, Backhaus TM, Hartung W, et al. Evaluation of a novel 7-joint ultrasound score in daily rheumatologic practice: a pilot project. Arthritis Rheum. (2009) 61:1194-201. doi: 10.1002/art.24646

59. Naredo E, Rodríguez M, Campos C, Rodríguez-Heredia JM, Medina JA, Giner E, et al. Validity, reproducibility, and responsiveness of a twelve-joint simplified power doppler ultrasonographic assessment of joint inflammation in rheumatoid arthritis. Arthritis Rheum. (2008) 59:515-22. doi: 10.1002/art.23529

60. Dale J, Stirling A, Zhang R, Purves D, Foley J, Sambrook M, et al. Targeting ultrasound remission in early rheumatoid arthritis: the results of the TaSER study, a randomised clinical trial. Ann Rheum Dis. (2016) 75:1043-50. doi: 10.1136/annrheumdis-2015-208941

61. Norvang V, Brinkmann GH, Yoshida K, Lillegraven S, Aga AB, Sexton J, et al. Achievement of remission in two early rheumatoid arthritis cohorts implementing different treat-to-target strategies. Arthritis Rheumatol. (2020) 72:1072-81. doi: 10.1002/art.41232

62. Mankia K, Briggs C, Emery P. How are rheumatologists managing anticyclic citrullinated peptide antibodies-positive patients who do not have arthritis? J Rheumatol. (2020) 47:305-6. doi: 10.3899/jrheum.190211

63. Mankia K, Emery P. Preclinical rheumatoid arthritis: progress toward prevention. Arthritis Rheumatol. (2016) 68:779-88. doi: 10.1002/art. 39603

64. Duquenne LPP, Mankia K, Nam J, Hunt L, Tan AL, Garcia-Montoya L, Emery P. At diagnosis of rheumatoid arthritis, at-risk patients followed in ccp + clinic showed milder disease activity than conventionally referred patients. Ann Rheum Dis. (2018) 77:A935. doi: 10.1136/annrheumdis-2018-eular.5704

65. Al-Laith M, Jasenecova M, Abraham S, Bosworth A, Bruce IN, Buckley $\mathrm{CD}$, et al. Arthritis prevention in the pre-clinical phase of RA with abatacept (the APIPPRA study): a multi-centre, randomised, double-blind, parallel-group, placebo-controlled clinical trial protocol. Trials. (2019) 20:429. doi: 10.1186/s13063-019-3403-7

66. Bos WH, Dijkmans BA, Boers M, van de Stadt RJ, van Schaardenburg D. Effect of dexamethasone on autoantibody levels and arthritis development in 
patients with arthralgia: a randomised trial. Ann Rheum Dis. (2010) 69:571-4. doi: 10.1136/ard.2008.105767

67. Strategy to Prevent the Onset of Clinically-Apparent Rheumatoid Arthritis (StopRA). (2020). Available online at: https://www.clinicaltrials.gov/ct2/show/ NCT02603146 (accessed April 1, 2020).

68. Van Boheemen SAT, Van Beers - Tas MH, Bos WH, Marsman D, Griep EN, Starman M, et al. Statins to prevent rheumatoid arthritis: inconclusive results of the STAPRA trial. Ann Rheum Dis. (2020) 79(Suppl. 1):1415-6. doi: 10.1136/annrheumdis-2020-eular.2805

69. Burgers LE, Allaart CF, Huizinga TWJ, van der Helm-van Mil AHM. Brief report: clinical trials aiming to prevent rheumatoid arthritis cannot detect prevention without adequate risk stratification: a trial of methotrexate versus placebo in undifferentiated arthritis as an example. Arthritis Rheumatol. (2017) 69:926-31. doi: 10.1002/art.40062

70. Falahee M, Finckh A, Raza K, Harrison M. Preferences of patients and at-risk individuals for preventive approaches to rheumatoid arthritis. Clin Ther. (2019) 41:1346-54. doi: 10.1016/j.clinthera.2019. 04.015
Conflict of Interest: KM reports personal fees from Abbvie, UCB, and Eli Lilly, outside the submitted work and research grants from BMS, Eli Lilly. PE reports consultant fees from BMS, AbbVie, Gilead, Galapagos, Lilly, MSD, Pfizer, Novartis, Roche, Samsung outside the submitted work and research grants from UCB, AbbVie, Lilly, Novartis, BMS, Pfizer, MSD, and Roche, outside the submitted work.

The remaining authors declare that the research was conducted in the absence of any commercial or financial relationships that could be construed as a potential conflict of interest.

Copyright (๑ 2020 Duquenne, Chowdhury, Mankia and Emery. This is an openaccess article distributed under the terms of the Creative Commons Attribution License (CC BY). The use, distribution or reproduction in other forums is permitted, provided the original author(s) and the copyright owner(s) are credited and that the original publication in this journal is cited, in accordance with accepted academic practice. No use, distribution or reproduction is permitted which does not comply with these terms. 